\title{
EL FUTURO DOMINICANO EN ESPAÑA: ALTO CAPITAL HUMANO, EMPODERAMIENTO Y RUPTURA CON LOS ESQUEMAS TRADICIONALES
}

\section{The Dominican future in Spain: high human capital, empowerment and breakage with the traditional schemes}

\section{Chiara Dello lacono}

Universidad de Salamanca. Espańa.

ORCID: 0000-0002-1963-5300

Recibido: 15/12/2018 • Aprobado: 17/1/2019

Cómo citar: Dello lacono, C. (2019). El futuro dominicano en españa: Alto capital humano, empoderamiento y ruptura con los esquemas tradicionales. Ciencia Y Sociedad, 44(1), 43-55. https://doi.org/10.22206/cys.2019.v44i1.pp43-55

\section{Resumen}

El trabajo analiza el proceso de empoderamiento de jóvenes mujeres dominicanas con alto capital humano emigradas a España. La hipótesis principal en la que se fundamenta el trabajo es que la variable migratoria puede ser considerada como clave del proceso de empoderamiento y, a la vez, como elemento de ruptura con la cultura "machista".

A partir de la teoría de Rowlands (1997) han sido analizados los elementos que permiten los cambios hacia el empoderamiento. Según el autor, el proceso se desarrolla a través de tres dimensiones: individual, colectiva y de las relaciones cercanas. Para cada una hay elementos positivos y negativos que afectan el proceso.

El trabajo se desarrolló mediante entrevistas con 18 jóvenes dominicanas que viven en Espańa. Las informaciones se obtuvieron a través del uso de la entrevista semiestructurada y de un cuestionario sociodemográfico.

Los resultados muestran que nos enfrentamos con una ola migratoria que es diferente a la anterior: una mezcla entre jóvenes con capital humano alto, otras en condiciones

\begin{abstract}
The work analyses the process of empowerment of young Dominican women with high human capital emigrated to Spain. The main hypothesis is that the migratory variable is on one side the key of the empowerment, and on the other, the element of rupture with the "macho" culture.

Starting from Rowlands's theory (1997), the elements that allow the changes towards the empowerment have been analysed. According to the author, the process is developed through three dimensions: individual, collective and close relationships. For each one there are positive and negative elements that can affect the process.

The work was developed through interviews with 18 young Dominican women living in Spain and the information obtained with the semi-structured interview and a sociodemographic questionnaire. The results show that we are faced with a migratory wave that is different from the others: a mix between young people with high human capital, others in economically unfavourable conditions and other single women with children.
\end{abstract}


económicamente desfavorables y madres solteras con hijos. Sí, es verdad que hay diferencias internas entre las mujeres, pero es cierto que a través de la migración estas consiguen crear proyectos de vida, empoderarse y romper con los esquemas tradicionales.

Palabras clave: jóvenes mujeres dominicanas; alto capital humano; migración; empoderamiento.

\section{Introducción}

El flujo migratorio dominicano hacia España es considerado uno de los más recientes y se ha consolidado a través de redes microsociales y cadenas migratorias activas (Valiente, 2003). Con relación a las razones de la migración, la elección del país de destino tiene que ver mucho con las condiciones que la sociedad de acogida ofrece: España cuenta y ha contado con políticas favorables a la migración proveniente de países latinoamericanos, entre estos República Dominicana.

Según los datos del $1^{\circ}$ enero de 2017 del Instituto Nacional de Estadística (INE), los inmigrantes que viven en España son 4,549,858. De estos, 69,630 son de origen dominicano, divididos en 38,587 mujeres y 31,043 hombres. A nivel europeo, como otros países del Mediterráneo, España ya presenta una cierta experiencia en el manejo de la migración, pero, junto con Grecia (15,6\%) e Italia (15,1\%) tiene un porcentaje muy bajo de población juvenil inmigrante entre 15 y 29 años, alcanzando esta solo el $15,1 \%$.

Los chicos y las chicas dominicanas presentes en el territorio español suman 23,140. Específicamente, en el grupo de edad de 15 a 29 años hay 12,635 mujeres.

Como se deduce de los datos, la migración femenina dominicana hacia España predomina sobre la masculina. Sin embargo, los pocos estudios que hay sobre el fenómeno migratorio de la mujer dominicana analizan solo la situación relacionada con el mercado laboral, con especial referencia
It is true that there are internal differences between women, but we can see that they manage to create life projects, empower themselves and break with the traditional schemes.

Keywords: Young Dominican women; high educational level; migration; empowerment.

al tema de las remesas, al mundo del trabajo doméstico y del cuidado, dejando de lado tanto el tema del empoderamiento como el de la migración juvenil.

A partir de los estudios teóricos sobre el empoderamiento, en este trabajo la hipótesis inicial es que la variable migratoria puede ser el elemento principal que, por un lado, promueve el proceso de empoderamiento y, por otro, representa la clave de ruptura con los esquemas tradicionales del machismo y del patriarcado. Estos esquemas aún son relevantes en la sociedad dominicana; por ejemplo, la disparidad de género en el mercado del trabajo (las mujeres dominicanas ganan un $44 \%$ menos que los hombres) y el nivel de ocupación y desempleo, donde las mujeres ocupan solo el 30\% de los cargos de mayor responsabilidad y registran una tasa de desempleo de un 23.1\%, más del doble del porcentaje de hombres, que es de un $9.7 \%$ (Lugo, 2012).

Claramente los ciclos migratorios cambian y con ellos las personas que emigran, a partir de sus circunstancias y sus perspectivas de vida. Sin duda, el proceso de empoderamiento y la situación de las jóvenes dominicanas que emigran hacia España no solo es interesante desde un punto de vista sociocultural, sino que también es un fenómeno que representa una apertura hacia nuevos horizontes de conocimiento.

En este contexto, las nuevas generaciones dominicanas quieren empoderarse a través de nuevos proyectos de vida y mediante la migración 
pueden conseguir un cambio a nivel de su situación y también de su condición de vida.

\section{Marco teórico}

En este trabajo de investigación se analiza el concepto de empoderamiento o, mejor dicho, el proceso de empoderamiento de las jóvenes mujeres dominicanas emigradas a España. Según la literatura de las Ciencias Sociales, existen diferentes definiciones sobre el empoderamiento femenino: Sharma (1991) lo define como un proceso orientado a cambiar la naturaleza y la dirección de las fuerzas sistémicas que marginan a las mujeres víctimas del sistema patriarcal y de la cultura machista; por su parte, Young (1997) señala que el empoderamiento consiste en facilitar el control de las mujeres sobre los procesos que afectan sus vidas.

Para nuestro estudio, nos parece más relevante el concepto de empoderamiento de Kabeer (2001): el desarrollo de la capacidad de las personas para tomar decisiones estratégicas de vida en un contexto en el que se les negó anteriormente. Este es un proceso que se adquiere a través del tiempo, haciendo cambios, e implica opciones efectuadas en base a ventajas y oportunidades reales.

Para algunos autores el empoderamiento es una variable latente alcanzable a través de ciertas características que varían dependiendo del grupo social y que no puede ser directamente observada o medida (Sinha, Jha $\&$ Negi, 2012); para otros, es un elemento que puede ser analizado desde una perspectiva cualitativa o cuantitativa (Gaye \& Shreyasi, 2011).

En el caso del empoderamiento femenino, ciertos estudios han demostrado que la mujer puede empoderarse en un área de vida, mientras no consigue hacerlo en otras (Malhotra \& Mather 1997; Kishor, 2000).

En el tiempo, el proceso de empoderamiento femenino ha sido analizado según variables diferentes: midiendo la autonomía (Dyson \& Moore, 1983; Basu \& Basu, 1991); el poder económico interno (Mason, 1998); el poder de la negociación (Beegle, Frankenberg \& Thomas, 2001; Hoddinott \& Haddad 1995; Quisumbing $\&$ de la Brière, 2000) o a través del patriarcado (Malhotra, 1995).

Los autores Hashemi Syed, Schuler \& Riley (1996), para analizar el empoderamiento de la mujer, utilizan ocho indicadores: movilidad, seguridad económica, capacidad de hacer una compra pequeña, capacidad de hacer una compra más grande, participación en decisiones, relativa libertad de dominación por la familia, participación política y conciencia jurídica.

Según Hugo (2000), en la base del proceso de empoderamiento hay un movimiento o proyecto migratorio, las características de las mujeres que emigran y el tipo de contexto. La incidencia de la migración de uno o más miembros de la familia influye sobre las funciones familiares: la ausencia, sea de manera permanente o temporal, influirá en la estructura familiar, tanto en las áreas de destino como en las de origen (Hugo, 2000).

Según Rowlands (1997), el empoderamiento, por un lado, incorpora la participación en la toma de decisiones y, por otro, es un proceso que lleva a las personas a percibirse como capaces y con derechos. El proceso de empoderamiento, según el autor, se analiza mediante tres dimensiones: personal, colectiva y de las relaciones cercanas. Por cada dimensión hay factores impulsadores e inhibidores.

1. En la primera dimensión, el empoderamiento se manifiesta con cambios individuales y los factores activadores del cambio son: el desarrollo de nuevos conocimientos, las actividades fuera del hogar, las actividades grupales y el tiempo para sí mismas. Estos son los agentes del poder de cambio sobre las propias habilidades, empezando por el autoestima, la dignidad y la capacidad de tomar decisiones. Por otro lado, los factores inhibidores están 
relacionados con el control masculino, el sistema patriarcal, la pobreza, la salud, la falta de control sobre la fertilidad, el cuidado y las obligaciones con los hijos (Rowlands, 1997).

2. La dimensión colectiva representa una perspectiva externa de empoderamiento, alcanzable mediante la acción grupal, la participación política y la capacidad colectiva. Los factores activadores son: los estímulos en el grupo, el desarrollo de liderazgo y la creación de redes de trabajo con otras organizaciones. Los factores que pueden inhibir el proceso son la falta de comunicación, una comunidad no cohesionada, la dependencia de los individuos claves y las políticas locales poco estables.

3. La última dimensión engloba los cambios hacia el empoderamiento que se obtienen a través de las relaciones cercanas. Las características a las que se hace referencia son las habilidades comunicativas, la capacidad de tomar decisiones propias y la capacidad de negociación para obtener el apoyo de los otros y defender los derechos de la mujer. Los factores que impulsan el cambio son: el conocimiento de los derechos propios, compartir problemas con otras mujeres y ser capaz de afrontar el tema de la desigualdad de género. Mientras los factores inhibidores son el machismo, la violencia familiar, la dependencia de la mujer y el control patriarcal.

\section{Metodología}

Las entrevistas se realizaron entre junio y octubre de 2016 en el territorio español, con un mayor enfoque en la comunidad de Madrid y la provincia de Salamanca. A través de las redes sociales, de Cáritas y Salamanca Acoge, se empezó a contactar a las chicas dominicanas que viven en el territorio español. La Cáritas Española es la Confederación oficial de las entidades de acción caritativa y social de la Iglesia Católica en España. Se ocupa de promover el desarrollo integral de las personas y los pueblos, especialmente de los más pobres y excluidos; se encuentra en ambas ciudades. Salamanca Acoge es una asociación sin ánimo de lucro que promueve el apoyo a las personas inmigrantes y refugiadas, así como la diversidad. Es una ONG reconocida como entidad de utilidad pública por el Ministerio del Interior y se encuentra solo en la ciudad de Salamanca. Ambas fueron elegidas por el trabajo diario que realizan para los inmigrantes; en nuestro caso, las mujeres dominicanas.

En total, las jóvenes entrevistadas fueron 18 y la metodología elegida fue la entrevista semiestructurada y el cuestionario sociodemográfico. Las entrevistas fueron grabadas en audio y transcritas para su posterior codificación y análisis. Los nombres han sido abreviados para garantizar su confidencialidad. Las mujeres entrevistadas han sido clasificadas en dos grupos de edad: el más joven de 14 a 24 ańos y uno intermedio con edades entre 25 y 38 años. En este último hay 3 chicas solteras con hijos.

La razón principal de la migración del grupo entrevistado es el estudio y, en segundo lugar, la reunificación familiar. La característica básica es el alto capital humano de las entrevistadas: la mayoría tiene título de estudio universitario y, en algunos casos, estudios universitarios superiores. Aunque nos enfrentamos con jóvenes con alto capital humano, hay diferencias internas particulares; por un lado, hay chicas que emigraron con una beca; mientras que, por otro, las hay sin recursos o que han recurrido a préstamos familiares.

En el primer grupo, la tasa de ocupación está relacionada con el elemento estudio, aunque hay una chica de 23 años que trabaja como profesora en una academia; por su parte, en el segundo, la mayoría tiene empleo público. En este grupo de edad la tasa de empleo en el servicio doméstico como cuidadora o limpiadora es muy bajo y solo una mujer está desempleada. 


\section{Análisis de los resultados}

Tomando el enfoquede Rowlandsysus dimensiones, se analizó el proceso de empoderamiento de las jóvenes dominicanas. Este análisis se presenta a continuación.

\section{Dimensión individual}

La primera dimensión analizada es la individual, a través de la cual los elementos que empujan al proceso de empoderamiento nacen de una serie de fuerzas internas de la mujer. Los factores que se desprenden de las entrevistas son el elemento estudio, los nuevos conocimientos, la independencia económica y la personal.

Gracias a la variable migratoria hay un cambio de prioridades y perspectivas; por lo tanto, el primer elemento positivo está relacionado con la migración por motivos de estudio y nuevos conocimientos. Está claro que nos enfrentamos con jóvenes que tienen alto capital humano, pero también es importante reconocer que es debido a un cambio generacional y de necesidades.

¿Sabes una cosa? No podía más. Vivir en casa era un infierno y alli las cosas son diferentes. ¿Me entiendes? Mi madre está pendiente de mi padre y de mi abuelo todo el rato. Ellos tienen la razón de todo. Creo que he tenido mucha suerte con la beca, sino estaría todavía viviendo con ellos".

\section{(S, 30 años, Pamplona)}

¿Por qué el elemento estudio podría empoderar a la mujer dominicana?

Según el análisis de las entrevistas, vivir en otro país, sobre todo en Europa, es un lujo y un objetivo que pocas mujeres, sobre todo jóvenes, pueden alcanzar. Antes que todo, es un gasto económico y, en segundo lugar, el mero hecho de ser una mujer joven que quiere salir de su tierra para estudiar en el extranjero es considerado un acto que tiene poco sentido y, a la vez, poco necesario. Aunque parte de la sociedad dominicana piense de esta manera, la mayoría de las mujeres realiza un gran esfuerzo para seguir estudiando fuera, formarse y obtener títulos académicos.

"Creo que el sistema educativo español es excelente! ¡Déjame decirte, realmente es excelente!

Nosotros en República Dominicana estamos en subdesarrollo. Aqui, desde que llegué, he visto los cambios".

( $R, 38$ años, Salamanca).

Analizando el elemento estudio salen algunos atributos de la cultura machista. En República Dominicana los hombres pueden hacer lo que quieran, eligiendo estudiar o trabajar; mientras que para la mujer la situación es diferente: ella está considerada solo en términos de ama de casa, cuidadora de la familia y de los niños. Estudiar o trabajar, sobre todo en otro país, no es una opción respetable.

"No quería más vivir con mis padres. Muchas veces me he sentido como si 'estuviera en una jaula', ipor las diferencias que habian entre mi hermano y yo! Claro, siendo hombre, puede hacer de todo. No tiene que preocuparse de nada. Por eso fue que cuando se me presentó la oportunidad de salir de República Dominicana, la cogí".

\section{(N, 24 años, Salamanca)}

El tema de la independencia económica representa el segundo elemento positivo hacia el empoderamiento. Para una mujer joven que ya ha vivido y vive tras las sombras del machismo y del patriarcado, el ser independiente económicamente es una victoria.

Esta nueva realidad no surge por una acción capitalista, sino por un cambio en la conciencia 
de la mujer dominicana, un punto de inflexión: la mujer puede vivir, tomar decisiones y buscarse la vida como mejor considere, sin una figura de autoridad que le diga cuándo, por qué y cómo hacerlo. Un buen ejemplo es el de A., una chica dominicana que desde 2006 vive en Salamanca:

"Sali de mi país gracias a una beca, me dieron esa oportunidad y me pareció estupendo. Alli no habría tenido la misma oportunidad, especialmente por el nivel socioeconómico de mi familia. Antes de llegar a España, en mi país, tenía dificultades por la pobreza de mi familia. Mis padres no podian hacer frente a los gastos de mis estudios. Vivo sola y ahora trabajo como profesora de inglés. Hice toda la formación académica en España y estoy super feliz".

Claramente las mujeres entrevistadas han logrado la independencia económica en diferentes fases y por diferentes razones. Analizando los datos, resulta que el "ser independiente" no está relacionado con la variable edad. Hay mujeres que emigraron para estudiar y mientras realizan sus estudios han decidido trabajar para enfrentarse a otras necesidades.

La independencia de las jóvenes dominicanas no solo es económica, sino un conjunto de elementos que una vez alcanzados permiten ganar una independencia personal y una tranquilidad en el sentido de un contexto social seguro.

Todas las mujeres entrevistadas han dicho que la vida en República Dominicana no es fácil: no se puede salir a la calle después de una cierta hora de la noche y hay que tener cuidado con la ropa que uno se pone. Para muchas llegar a España y salir con las amigas de noche ha sido una cosa nunca vista antes. La tranquilidad de poder salir sola de casa o regresar después de una determinada hora puede ser visto como algo frívolo, pero para ellas es una victoria y un cambio individual importante.

"Tengo mi familia en República Dominicana pero mi madre quiere venirse a España por el tema que allí hay mucha delincuencia. No puedes ir tranquila por las calles".

\section{(A, 23 años, Salamanca)}

Mediante el análisis de las entrevistas, surge que no solo hay elementos positivos o negativos del cambio de vida, sino también transversales: elementos que permiten y pueden obstaculizar a la vez el proceso de empoderamiento. Uno de estos, según las respuestas de las chicas entrevistadas, es la presencia de los hijos. En el grupo de edad de 25 a 39 años, hay 3 chicas solteras con hijos. Vamos a ver las historias.

"Cuando llegué me quedé en Madrid, luego me fui para Salamanca, donde comparto piso con otras chicas dominicanas. Mi hijo tiene 6 años y vive en Madrid con su tía, lo veo muy poco. La reunificación es vital, yo necesito estar con él, pero ir a Madrid es muy caro. Ahora lucho porque él tenga un futuro mejor".

\section{(M., 26 años, madre soltera)}

"Creo que el empoderamiento de la mujer en la vida laboral puede afectar la educación de los hijos, pero es justo asi. Buscar un equilibrio es muy complicado".

\section{(F, 26 años, Salamanca)}

Otras se sienten culpables únicamente por el hecho de querer estudiar y regalar a sus hijos un futuro mejor fuera de su país. Por ejemplo, la historia de R., de 38 años. Ella trabajaba en República Dominicana como enfermera, pero quería ampliar sus estudios en psicología. Así comenzó el proceso para obtener unos documentos especiales que le permitieran estudiar un máster y trabajar a la vez. Tiene 3 hijos que mantener y es madre soltera.

"Mis hijos tienen: el mayor, 23 años; la segunda, 15; y el menor, 13. El mayor está en Estados Unidos. Los menores normalmente viven conmigo, pero desde que me fui a España, 
los dejé con una cuidadora en mi casa. Quiero volver a R.D., pero ahora no puedo".

La separación de la familia es otro elemento transversal encontrado en el análisis. Por un lado, hay mujeres que finalmente pueden hacer lo que quieren dejando atrás los aspectos de la cultura machista; mientras hay otras que, aunque sufran el machismo de su pareja o la imposición de su padre, sienten la falta de ese estilo de vida y se encuentran perdidas sin ellos.

"He perdido el vínculo familiar, he perdido mi zona de confort".

\section{(A, 26 años, Salamanca)}

"He perdido el contacto con mi familia, lo llamo "desmembramiento personal". Llegando a España tan joven, fue bastante duro y complejo enfrentarme con la realidad de las cosas. Estaba sola"

\section{(L,23 años, Salamanca)}

Cuando se encuentran elementos negativos en una dimensión, en este caso la individual, significa que algunas dinámicas no pueden perfilarse fácilmente. Los elementos negativos que se encontraron son los lazos con la República Dominicana.

Las respuestas a la pregunta: ¿qué has perdido llegando a España? son diferentes. Por un lado, hay mujeres que sufren por haber perdido las afecciones primarias como la familia, la convivencia con los hijos y los amigos; por otro, viene reevaluado el sentido común de nacionalidad. Según el estudio, algunas de las jóvenes entrevistadas se sentían y se sienten solas desde que eligieron migrar a España.

"Cuando llegué, tenía que vivir en un internado de monjas y todo fue complicado. Hasta la comida no me gustaba y me faltaban muchas cosas".

( $G, 26$ años, Madrid)
"Dado que estoy estudiando no puedo vivir con mi hijo, no tengo dónde dejarlo. Entonces está en Madrid con una tía que me lo cuida".

( $M, 26$ años, madre soltera, Salamanca)

"He perdido mi centro terapéutico donde estuve trabajando como psicóloga clínica".

( $R, 25$ años, Salamanca)

\section{Dimensión colectiva}

Según la dimensión colectiva, los hipotéticos cambios para el empoderamiento se logran a través de la acción grupal: cambiando la perspectiva del sujeto desde el "yo" hacia el "nosotros". El nivel de asociación de la mujer dominicana en el territorio español y la consiguiente acción colectiva fueron analizadas a través del nivel de conocimiento de Cáritas, las asociaciones universitarias y las asociaciones de dominicanos que viven en España.

Con relación a la acción grupal, el fenómeno de la guetización de las generaciones latinas que viven en España (sobre todo la dominicana) es muy fuerte ${ }^{1}$.

En este trabajo el tema de la guetización no ha sido tratado, pero, preguntando qué significaba y si las jóvenes eran conscientes de él, resultó que se trata de un fenómeno de asociación masculina que no solo ocurre en las grandes ciudades, sino también en las pequeñas realidades.

1. Telemadrid, 16/03/16 h.17.51 www.telemadrid.es Entrevista a Aníbal de Castro, embajador de la República Dominicana en España. Al ser preguntado por la proliferación de bandas latinas, dice: "En la Comunidad de Madrid hay unos 28.000 dominicanos y otro tanto de espańoles de origen dominicano. Si además debemos tener en cuenta que hay cerca de un millón de personas de la comunidad iberoamericana, pues los integrantes de las bandas son una parte muy pequeńa de la población, lo que no quiere decir que no sea un problema. Yo creo que hay razones sociales, culturales, también otro factor podría ser la falta de oportunidad, el paro entre los jóvenes... es un problema muy complejo". 
A diferencia de los hombres, parece que las mujeres están más abiertas a la integración en el territorio, sin dar importancia a la nacionalidad de la persona. Desde el punto de vista de las relaciones sociales, como se ha señalado antes, las mujeres no tienen preferencia.

A través de los datos resulta que las chicas estudiantes se relacionan más fácilmente con otras estudiantes, mientras otras del grupo (25-39 años) prefieren relacionarse con las dominicanas, para sentirse como "en casa".

Sobre la base de las respuestas, el fenómeno de asociación de la mujer dominicana se relaciona con la edad y la variable empleo.

Desde un punto de vista general, las asociaciones de voluntarios tienen un mayor impacto en las mujeres mayores, de 25 a 39 años. Todas las jóvenes dominicanas entrevistadas conocen o han tratado con asociaciones de voluntarios del territorio. Hay alguna que también es voluntaria de Cáritas:

"Estas instituciones me parecen muy buenas y creo que hacen un gran aporte a la población de bajos recursos".

(P, 33 años, cuidadora de mayores)

Otro elemento positivo es la relación entre las chicas dominicanas y la población de acogida. Resulta que la mayoría de las entrevistadas fue bien recibida por parte de los españoles.

"He sido muy bien recibida por la población española y tengo muchos amigos españoles".

\section{(S, 38 años, Salamanca)}

"Si y no! He sido más o menos bien recibida. Siempre hay pro y contra. Tengo muchos amigos españoles y también estoy conociendo un chico español".

(G, 26 años, Madrid)
El primer elemento negativo de la dimensión colectiva es la falta de participación política de las jóvenes. Ninguna de las chicas entrevistadas está interesada en el tema político. Este tema no se refiere a las cuestiones relacionadas con las elecciones o prácticas similares, sino a la política entendida como conjunto de derechos y deberes que cada ciudadano, migrante o no, debe conocer y respetar: la participación en la vida política. Ninguna da importancia a la cuestión, aunque todas vivan en el territorio español y algunas de ellas tengan la nacionalidad o estén a punto de conseguirla.

"No he votado porque todavía no tengo la nacionalidad y la verdad es que no estoy interesada".

\section{(G, 26 años, Madrid)}

Otros elementos negativos que detienen el proceso de empoderamiento son los prejuicios y los estereotipos. Estos, detrás de la discriminación de género o por parte de la sociedad de acogida, afectan de modo negativo y causan daños a las mujeres.

En general, la mujer dominicana, a pesar de ser una mujer fuerte y luchadora, muchas veces, sobre todo si es joven y en pleno proceso de empoderamiento, resulta fuertemente afectada:

"Creo que la población española tiene prejuicios contra los dominicanos, especialmente contra las mujeres. Te voy a contar algo muy intimo: cuando comencé a salir con un chico, él quería venirse a la cama conmigo, yo me sentí mal. No estoy acostumbrada a eso. Hemos comenzado a hablar y le dije que eso no es normal en mi pais. El me dijo: "fijate que yo pensaba que fuese distinto". Ellos piensan que las mujeres dominicanas son calientes. Muchos te ven como objeto sexual y me molesta"

( $N, 24$ años, Salamanca)

"Alguien me ha tratado de manera diferente por ser dominicana. Cuando estuve haciendo la F. 
P. en Salamanca, especialmente el último año, me senti muy discriminada porque en el curso habia racistas".

(M, 26 años, Madrid)

"En general fui bien recibida, aunque siempre hay estereotipos de gente que por la calle dice: "las latinas son muy contentas y divertidas".

( $R, 23$ años, Salamanca)

\section{Dimensión de las relaciones cercanas}

Uno de los elementos positivos de la dimensión de las relaciones cercanas es el reconocimiento del papel de la mujer dominicana. Este es uno de los temas más interesantes y más delicados tratado en las entrevistas.

A la pregunta: ¿Qué opinas acerca del papel de la mujer dominicana? En general, como si se tratara de una sola voz, la respuesta fue: “Estamos en lucha! Es un papel totalmente desvalorizado, pero estamos en el medio de un cambio".

El tema del machismo en la cultura dominicana, como en la mayoría de las culturas latinas, no es nuevo y, dado que está tan anclado en la estructura societaria, tal vez pasa inobservado:

"Mi pais, como casi todos los paises de América Latina, tiene una cultura machista. La mujer desde pequeña vive un papel degradante: tiene que estar siempre en casa y el hombre es el que trabaja. Solo por ser hombre tiene el derecho a no hacer nada en casa".

\section{(V, 26 años, Salamanca)}

Hasta ahora, los tentativos de cambio de la mujer dominicana han sido pocos y de escasa relevancia. Hace ya unos ańos, gracias a elementos como el estudio, la formación profesional y unas instituciones como la universidad, las dominicanas han empezado a pensar críticamente. De hecho, las perspectivas están empezando a ser diferentes y las jóvenes quieren encontrar su lugar en el mundo.

El tema del machismo es bastante conocido, así que, desde luego, dar espacio a la voz de las entrevistadas es más importante.

"La mujer en mi pais tiene un papel que va creciendo. Todavía vivimos en una sociedad donde la cultura machista es predominante, pero la mujer está finalmente saliendo de los esquemas del modelo patriarcal. Es algo que va cambiando y creciendo con el tiempo. Creo que no solo la mujer tiene que cambiar, sino también la ley".

\section{(M, 22 años, Salamanca)}

"La mujer dominicana ha superado bastantes barreras en la sociedad: ha ido creciendo en el campo laboral, académico, gerencial, empresarial y social. Todavía falta mucho para mejorar, sobre todo el tema de la exclusión femenina en unos empleos y los abusos de parejas entre otros".

( $R, 30$ años, Pamplona)

De acuerdo con la pregunta: ¿Tus derechos como mujer tienen más sentido en España u opinas que tienen la misma entidad que en R. D.? La mayoría de las mujeres respondió que sus derechos como mujer son más relevantes en España.

"El sentido de los derechos de la mujer entre España y República Dominicana es completamente diferente. La República Dominicana tiene una cultura muy machista. Por ejemplo: ya el venir aqui desde mi pais ha supuesto un gran enriquecimiento. Muchas cosas que hay alli no son correctas $y$, de hecho, los derechos de ser mujer tienen mayor validez aqui. La mujer alli es inferior. Es como una norma todo eso y todo lo que se enfrenta con la norma es un shock. Aqui la historia es diferente".

( $A, 23$ años, Salamanca) 
Desde luego, para ellas que viven un modelo de vida completamente diferente del que se vive en países europeos, parece que la cultura machista no está tan presente en el territorio español. Pero, aunque con diferente forma, la hay.

"Lo que marca la diferencia es la cultura, la práctica. ¡Resulta que yo tengo que valorar mis derechos! ¡Eso es mi trabajo!”

\section{(N, 24 años, Salamanca)}

Por un lado, hay mujeres que luchan para garantizar sus derechos y libertades; por otro, hay dinámicas particulares donde la mujer sabe que tiene que cambiar algo, pero no quiere, porque es así y es un dogma a priori.

Claramente es un tema delicado, pero son muchas las mujeres dominicanas que todavía no se dan cuenta que la que viven no es vida, ni siquiera libertad.

En algunas de las entrevistas se desprende que la existencia del machismo está relacionada con el rol de la mujer $y$, aunque la mujer dominicana haya reconocido su papel en la sociedad, es evidente que la influencia del hombre sigue siendo muy fuerte. El problema es que, si la mujer no entra en la dinámica del cambio, es complicado que el proceso de empoderamiento comience.

"La mujer quiere ser más activa. A la vez, creo que la mujer es el primer agente del machismo. ¡Es ella que crea a los machistas! iLa primera machista es la mujer! ;Es importante que la mujer vaya a la universidad, ique descubra todo un mundo! Que se sienta inspirada por ejemplos de otras mujeres que quieren cambiar las cosas. La decisión de luchar llega por un estímulo externo".

\section{(N, 24 años, Salamanca)}

"En la cultura dominicana todavía se ve el machismo. Nosotros somos machistas, esa es la realidad. Es una cultura machista, pero nosotros somos culpables de este proceso. Las mujeres somos las que criamos: los hombres están en la calle, nosotras en casa, pero no es siempre asi. ¿Debiamos que cambiar la educación familiar!

\section{(F,38 años, Salamanca)}

En las relaciones cercanas, desde un punto de vista general, el elemento que bloquea el cambio de vida de las jóvenes es el fenómeno de la discriminación. Aunque sea complicado empezar el proceso de cambio interno, grupal y de las relaciones cercanas, cuando hay elementos como la discriminación, es difícil continuar.

Algunas de las entrevistadas piensan que, en general, hay muchas personas que tienen estereotipos sobre las mujeres dominicanas; no es fácil que estas últimas cambien de opinión, pero a veces sucede. Sucede cuando empiezan a relacionarse con la comunidad dominicana y ven que no todas son iguales y que no es justo opinar sobre algo sobre lo cual no hay conocimiento previo.

Diferente es la situación de las personas que discriminan: no cambian y empeoran con el tiempo. La mayoría de las entrevistadas se define como "luchadora"; una mujer que ha decidido emigrar sola, afrontar la vida y vivir sin nadie; pero, cuando viene discriminada, ya sea por la tierra de origen o el país de destino, se siente frágil, abandonada y muchas veces se da por vencida.

"Nunca he sufrido la discriminación, pero una señora por la calle me dijo: hay chicas como tú (latinas) que son muy exóticas y que 'van' con personas mayores. Normalmente, por una mala acción de un dominicano, puede llegar a sufrir todo el colectivo. La idea es que las chicas latinas son todas putas y alegres y los chicos son todos delincuentes. Estos son estereotipos. Soy latina, pero no me gusta la bachata, ni siquiera la escucho en la radio".

( $A, 22$ años, Salamanca) 


\section{Conclusiones}

Al día de hoy, la investigación cualitativa $y$ cuantitativa sobre el empoderamiento de la mujer dominicana resulta ser casi nula o inexistente. En las ciencias sociales, la mayoría de los estudios refleja la situación laboral de las mujeres mayores dominicanas, sin mirar los cambios generacionales y los nuevos proyectos migratorios.

Gracias a los cambios sociales, las redes entre las instituciones y las organizaciones internacionales, parece que la migración juvenil desde República Dominicana hacia España empieza a ser más fuerte y más presente.

Los nuevos flujos se componen de jóvenes mujeres con alto capital humano, tal vez sin recursos y algunas solteras con hijos. El hilo conductor entre estos mundos diferentes es el deseo de empoderarse, las ganas de poder conquistar un rol en la sociedad y la ruptura con los esquemas tradicionales del machismo.

Aplicando la teoría de Rowlands (1997) al grupo de las 18 jóvenes dominicanas que viven en España y que fueron entrevistadas para este estudio, se puede comprobar que la migración no solo es el núcleo de los cambios hacia el proceso de empoderamiento, sino que es el sinónimo de ruptura con el modelo patriarcal y la cultura machista. En todas las entrevistas las chicas han dicho que gracias a la migración han tenido la oportunidad de cambiar y empezar el proceso de empoderamiento.

En las tres dimensiones analizadas se produjeron cambios positivos, negativos y transversales. Los positivos son un claro ejemplo de que el proceso de empoderamiento, de alguna manera, ya empezó. Igualmente importantes son los negativos, porque ayudaron a observar los posibles elementos sobre los que las jóvenes dominicanas tienen que "trabajar"; mientras los transversales son particulares, porque ni permiten el desarrollo del proceso ni lo obstaculizan.
La primera dimensión del estudio es la individual, donde los cambios que se verifican son de tipo personal. Estos cambios son propios de las mujeres que empiezan a mover sus primeros pasos dentro del proceso de empoderamiento. Nos enfrentamos con elementos como el estudio y los nuevos conocimientos, la independencia económica y la tranquilidad ganada a través de un contexto social seguro. Dichos elementos se relacionan todavía con la cultura machista, pero gracias a la migración empiezan a tomar una forma diferente.

Mediante las entrevistas analizadas, hemos encontrado que el elemento que permite el cambio general hacia el proceso de empoderamiento es la variable estudio. Este no solo está relacionado con las dominicanas con alto capital humano, sino con la necesidad de estudiar para poder cambiar de vida. No todas las jóvenes llegaron a España con título de estudio o con recursos para empezar a estudiar, pero consiguieron hacerlo.

Como se desprende de las entrevistas, el deseo de empoderarse y obtener un título es contrastado por la presencia de los hijos. En el grupo de edad de 25 a 39 años hay 3 chicas solteras con hijos y, como se ha demostrado en este análisis, no es fácil dejarlos solos. Pero, la realidad es diferente y para ellas no hay otra forma, sino esta, para lograr un futuro mejor.

En la dimensión colectiva el proceso de cambio pasa a través de la acción grupal. Resulta que las jóvenes tienen una buena relación con las asociaciones de voluntariado y con la población de acogida, aunque la presencia de estereotipos contra la mujer dominicana produce en ella misma temores que ralentizan el proceso.

El tema de los prejuicios aparece en la mayoría de las entrevistas y parece que de verdad limita el desarrollo del proceso de empoderamiento. En la dimensión de las relaciones cercanas, resulta que las jóvenes dominicanas están intentando reconocer su rol en la sociedad mediante la capacidad de tomar 
decisiones propias, las habilidades comunicativas y el poder de negociación. La mujer no solo es ama de casa o cuidadora de los hijos, ella debe tener los mismos derechos que el hombre eligiendo su vida.

$\mathrm{Si}$, por un lado, el proceso de empoderamiento de las relaciones cercanas se desarrolla mediante el reconocimiento de los derechos y enfrentando las desigualdades de género; por otro, hay factores que lo inhiben: el machismo, la violencia familiar, la dependencia de la mujer, el control patriarcal. El modelo patriarcal y la cultura machista son un dogma a priori inculcados en la sociedad y mediante las entrevistas se nota que el proceso de empoderamiento de las chicas dominicanas es lento, pero no imposible: el deseo de autorrealización y de lucha de los nuevos flujos hacia España es fuerte.

Se concluye que, a pesar de un papel en total construcción y con carencias de recursos, las jóvenes dominicanas, mediante la migración y gracias al elemento estudio, están desarrollando habilidades y creando sus proyectos de vida autónoma, lo que nos hace presagiar un proceso de empoderamiento.

\section{Referencias}

Basu, A. M. \& Basu, K. (1991). Women's economic roles and child survival: the case of India. Health Transition Review, 1(1), 83-101.

Beegle, K.; Frankenberg, E. \& Thomas, D. (2001). Bargaining power within couples and use of prenatal and delivery care in Indonesia. Studies in Family Planning, 32(2), 130-146.

Dyson, T. \& Moore, M. (1983). On Kinship Structure, Female Autonomy and Demographic Behaviour in India. Population and Development Review, Population Council, 9(1), 35-60.

Gaye, A. \& Shreyasi J. (2011). Measuring Women's empowerment through migration, Diversities, 13(1).

Hashemi Syed, M.; Schuler, S. R. \& Riley, P. A. (1996). "Rural Credit Programs and Women's
Empowerment in Bangladesh." World Development, 24(4), 635-653.

Hoddinott, J. \& Haddad, L. (1995). "Does Female Income Share Influence Household Expenditures? Evidence from Cote d'Ivoire." Oxford Bulletin of Economics and Statistics, 57(1), 77-96.

Hugo, G. (2000). "Migration and Women's Empowerment." En Harriet B. Presser \& Gita Sen eds., Women's Empowerment and Demographic Processes: Moving Beyond Cairo, Oxford: Oxford University Press, 287-317.

Kabeer,N. (2001). "Reflectionson the Measurement of Women's Empowerment". En Discussing Women's Empowerment-Theory and Practice. Sida Studies 3. Stockholm: Novum Grafiska AB.

Kishor, S. (2000). Empowerment of women in Egypt and links to the survival and health of their infants. En Presser H.B. \& Sen G. (eds). Women's Empowerment and Demographic Processes, New York: Oxford University Press.

Lugo, G. "The Dominican Republic's epidemic of domestic violence," 2012, The Guardian, Recuperado de https://www.theguardian.com

Malhotra A., Vanneman, R., \& Kishor, S. (1995). "Fertility, Dimensions of Patriarchy, and Development in India." Population and Development Review, 21(2), 281-305.

Malhotra, A. \& Mather, M. (1997). Do schooling and work empower women in developing countries? Gender and domestic decisions in Sri Lanka, Sociological forum, 12(4), 599-630.

Mason, K. (1998). "Wives' Economic Decisionmaking Power in the Family: Five Asian Countries." The Changing Family in Comparative Perspective: Asia and the United States. Karen Oppenheim Mason, ed. Honolulu: East-West Center, 105-133.

Quisumbing, A. R. \& de la Brière, B. (2000). Women's Assets and Intrahousehold Allocation in 
Rural Bangladesh: Testing Measures of Bargaining Power. Discussion Paper-Food Consumption and Nutrition Division, International Food Policy Research Institute, 86, p41.

Rowlands, J. (1997) "Empoderamiento y mujeres rurales en Honduras: un modelo para el desarrollo". En León M., (Ed) Poder y empoderamiento de las mujeres, Bogotá: Tercer Mundo S. A.

Sharma, K. (1991-1992). "Grassroots organizations and women's empowerment: Some issues in the contemporary debate", Samya Shakti, 6, 28-43.

Sinha, B.; Jha, S. \& Singh Negi N. (2012). Migration and empowerment: the experience of women in households in India where migration of a husband has occurred, Journal of Gender Studies, 21, 1, 61-76.

Valiente, J. M. R. (2003). La migración dominicana hacia España, factores, evolución y desarrollo, Revue européenne des migrations internationales, 19(1).

Young, K. (1997). "El potencial transformador en las necesidades prácticas: empoderamiento colectivo y el proceso de planificación". En León M., (Ed.), Poder y empoderamiento de las mujeres, Bogotá: Tercer Mundo S. A.

\section{Datos de filiación}

Chiara Dello Iacono. Máster en Servicio Social y Políticas Sociales. Actualmente cursa un Doctorado en Sociología en la Universidad de Salamanca (España). Su tema de investigación se refiere a los procesos de integración de los inmigrantes con particular énfasis en la población de dominicanos en España. Correo electrónico: delloiaconochiara@gmail.com 